\title{
Évolution du potentiel hydrique et du système racinaire de jeunes plants de cèdre, pin laricio de Corse et pin noir plantés à l'automne et au printemps
}

\author{
G. AUSSENAC et M. EL NOUR \\ I.N.R.A., Station de Sylviculture et de Production, \\ Centre de Recherches forestières de Nancy, Champentoux, F 54280 Seichamps
}

\begin{abstract}
Résumé
Les auteurs ont suivi, après plantations à racines nues en automne et au printemps, l'évolution du potentiel hydrique de base et l'état de régénération du système racinaire de plants de cèdre, de pin laricio de Corse et de pin noir d'Autriche. Deux traitements ont été considérés : traitement I (témoin) : les plants ont conservé leurs extrémités racinaires blanches, traitement II : toutes les racines blanches ont été excisées. Les observations effectuées montrent que quel que soit le traitement, les plants pourvus de racines blanches au moment de la mesure, ont un potentiel hydrique de base significativement plus élevé que ceux qui en sont dépourvus. Lorsque le potentiel de base était inférieur à $-1,5 \mathrm{MPa}$, les plants ne présentaient pas de racines blanches. L'étude de la répartition des plants, par classe de potenticl hydrique, montre que le pin laricio de Corse, contrairement aux deux autres espèces, a des difficultés à maintenir un état hydrique élevé malgré une plantation préservant les extrémités racinaires. L'excision des racines blanches a eu, pour conséquence. des taux de débourrement faibles pour le cèdre et le pin noir d'Autriche, et nuls pour le pin laricio de Corse. Globalement, des trois espèces étudićes, le pin laricio de Corse a été celle qui a été la plus perturbée par la plantation à racines nues.
\end{abstract}

Motx clés: Potentiel hydrique, régénération racine, plantation.

\section{Introduction}

Pour certaines espèces, la plantation à racines nues s'accompagne d'une crise de transplantation et d'une mortalité quelquefois importante. Ces phénomènes sont liés aux traumatismes subis par les racines au moment et à la suite de l'extraction des plants en pépinière. On peut penser que ces phénomènes sont en relation directe avec les possibilités de régénération et de croissance de nouvelles racines (ARBEZ, 1971) qui doivent conditionner la capacité du système racinaire d'extraire l'eau du sol permettant aux plants d'avoir un bon état hydrique interne compatible avec le déroulement des fonctions telles que la photosynthèse et la croissance de la partie aérienne.

Dans ce travail, on a étudié l'évolution du système racinaire et de l'état hydrique des plants, afin de préciser le rôle des nouvelles racines régénérées après plantation 
en automne et au printemps. Ces études ont été réalisées sur cèdre (Cedrus atlantica Manetti), pin laricio de Corse (Pintus nigra Arn. ssp. Laricio Poiret var. corsicana) et pin noir d'Autriche (Pintus nigra Arn. ssp. nigricans Host.). On sait que les deux premières espèces sont réputées subir une mortalité importante lors de plantations à racines nues.

\section{Matériel et méthodes}

On a suivi en parallèle, après plantation en automne et au printemps, l'évolution de l'état hydrique des plants et de leur système racinaire.

Sur l'ensemble des plants considérés "', les racines ont été coupées à $25 \mathrm{~cm}$ du collet. Deux lots de plants ont alors été constitués :

- le lot I $(\bullet)$ pour lequel on a gardé toutes les extrémités racinaires blanches : excisées.

- le lot II (O) pour lequel les extrémités racinaires blanches ont toutes été

L'expérimentation a été réalisée en pépinière au Centre National de Recherches Forestières, près de Nancy. Dans tous les cas, les plants ont été arrosés à la plantation. Pour ce qui concerne les plantations de fin d'été et d'automne, le tableau 1 donne l'âge et le nombre de plants utilisés, les dates de plantations.

\section{Tableau 1}

Dates de plantation en automne, âge et nombre de plants utilisés pour les deux traitements. Date of plantations in autumn, age and number of seedlings utilized.

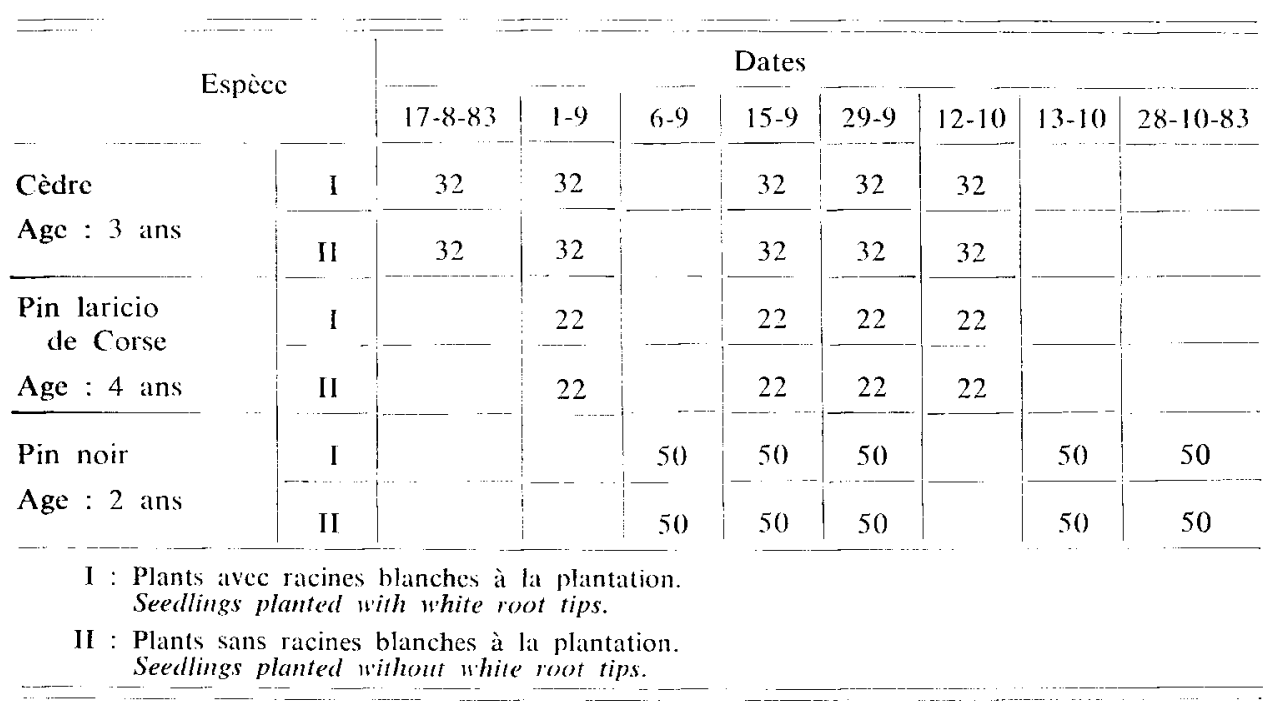

(1) Afin de constituer des lots de plants de taille voisine, on a éliminé de la population initiale les plants les plus petits et les plants les plus grands. 
Au printemps, les plantations ont été faites le $1^{\text {tr }}$ avril 1983 et le 17 mai 1983 ; elles n'ont concerné que le cèdre (50 plants âgés de 2 ans) et le pin laricio de Corse (50 plants âgés de 3 ans).

Après chaque plantation, au cours des 2 mois suivants, tous les 15 jours trois plants ont été prélevés, afin de mesurer le potentiel hydrique de base (mesure de fin de nuit) et d'observer l'état du système racinaire. Le potentiel hydrique (- MPa) (2) a été mesuré à l’aide d'une bombe de Scholander.

\section{Résultats}

\subsection{Potentiel hydrique et régénération des racines en automne}

Le potentiel de base est une mesure du potentiel hydrique de l'arbre au moment le plus favorable de la journée (fin de la nuit) : recharge maximum en eau, transpiration réduite. Si le système racinaire est bien développé, le potentiel de base est proche du potentiel hydrique du sol (Aussenac et al., 1984). Dans cette expérimentation, les arbres étaient fréquemment arrosés, afin de maintenir le sol proche de la capacité au champ (potentiel hydrique voisin de - 0,1 - - 0,2 MPa). Des écarts élevés à ces potentiels hydriques reflètent l'importance du stress subi par les plants, en raison de leur incapacité à mobiliser l'eau du sol. Dans les conditions de l'expérimentation, le niveau - 0,5 MPa peut être considéré comme le seuil de référence en dessous duquel les plants sont en déséquilibre avec l'eau du sol.

\subsection{Cas du cèdre (fig. 1)}

Pour ce qui concerne la plantation du 17 août 1983, l'état hydrique des plants reste bon (supérieur à $-0,35 \mathrm{MPa}$ ) pour les 2 lots considérés. Le système racinaire s'est bien régénéré, même chez les plants du traitement II (O) qui ont eu toutes ícurs racines blanches exciscés. Pour la plantation du $1^{\text {w }}$ septembre 1983 , les plants du traitement $I(\bullet)$ ont un meilleur état hydrique que ceux du traitement II $(O)$. Cette situation va de pair avec une présence quasi générale de racines blanches pour le premier traitement, alors qu'il n'en est pas de même pour les plants ayant cu toutes leurs racines excisées.

A la suite de la plantation du 15 septembre 1983, l'état hydrique des plants du traitement 1 est nettement meilleur que celui des plants du lot II chez lequel on observe une grande hétérogénéité des potentiels hydriques. Ici encore, ces potentiels faibles vont de pair avec l'absence de nouvelles racines blanches.

Après les plantations du 29 septembre 1983 et du 12 octobre 1983, on constate à nouveau que l'état hydrique des plants du traitement II est très variable d'un plant à un autre ; alors qu'il n'en est pas de même pour le traitement I. Le 24 novembre 1983 et le 9 décembre 1983, la baisse des potentiels hydriques s'explique par des gelées importantes à $\mathrm{cc}$ moment là. Cette baisse est cependant plus importante pour les plants du traitement II. Le 9 décembre 1983, les plants examinés ne présentaient plus de racines blanches, probablement à cause du refroidissement du sol.

(2) $1 \mathrm{MPa}=10$ bars. 


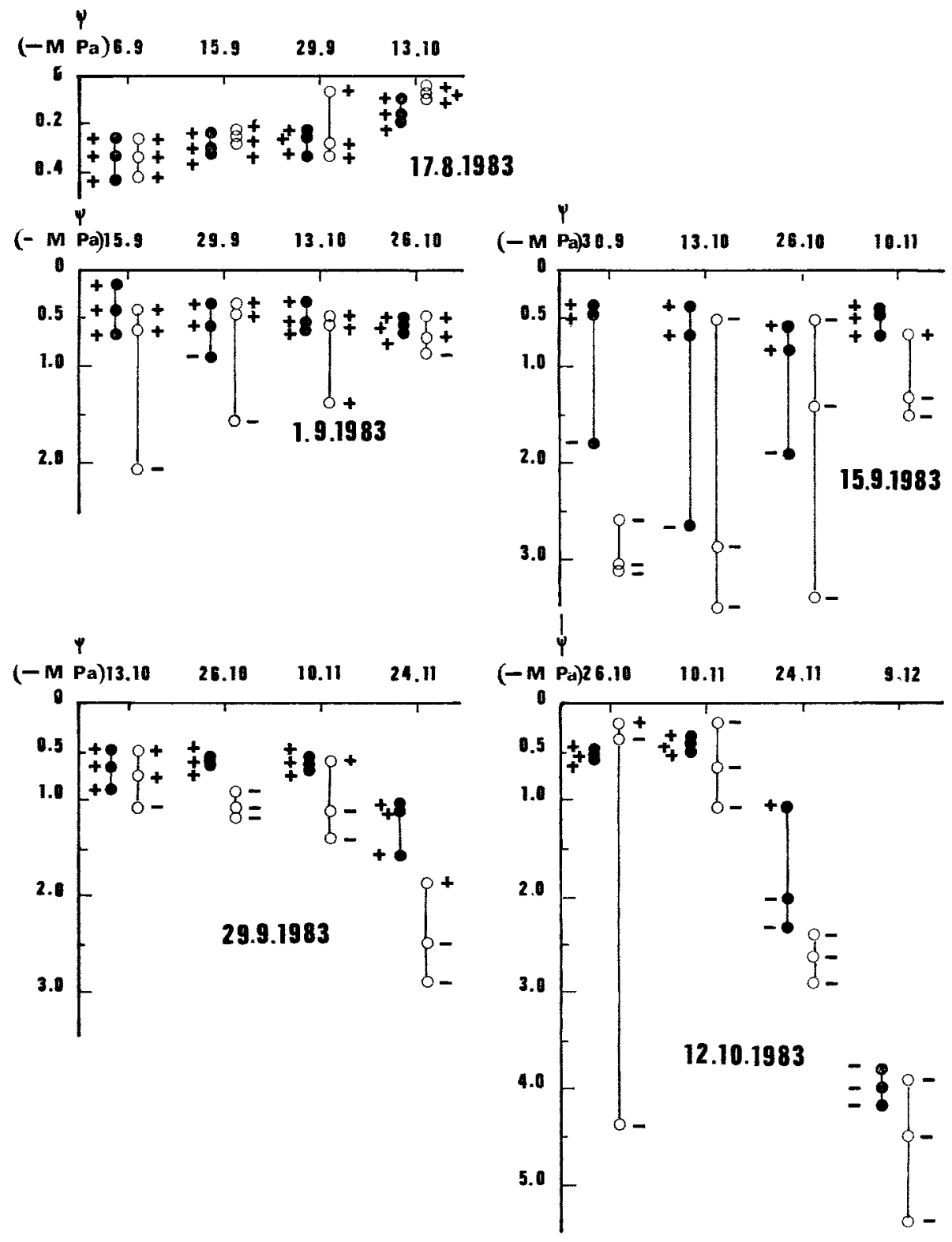

FIG. 1

Evolution, après plantations en automne, du potentiel hydrique de base (-MPa), présence $(+)$ ou absence $(-)$ de racines blanches pour des plants de cèdre plantés avec racines blanches $(\bullet)$ et sans racines blanches $(0)$.

Predawn potential after plantation in autumn, presence $(+)$ or absence $(-)$ of white root tips for Cedrus atlantica planted with white root tips $(\bullet)$ and without white root tips $(0)$. 
Les taux de débourrement et de survie des plants des différentes plantations ont été constatés en juin 1984. Les taux de débourrement (tabl. 2) des plants du traitement II (racines excisées) sont d'une façon générale inférieurs à ceux des plants du traitement I. Pour ce dernier traitement, les taux de débourrement sont les plus bas pour les plantations du $1^{\text {rr }}$ septembre et du 15 septembre 1983 qui ont aussi les taux de survie les plus bas.

\section{TABleau 2}

Taux de débourreme'nt (p. 100) des plants de cèdre, pin laricio de Corse' at pin noir d'Autriche des différentes plantations d'automne.

Rate of budbreak (p. 100) of seedlings of Cedrus atlantica, Corsican pine, Austrian pine after the altumn plamtations.

\begin{tabular}{|c|c|c|c|c|c|c|}
\hline \multirow{3}{*}{ Date plantation } & \multicolumn{6}{|c|}{ Espèce } \\
\hline & \multicolumn{2}{|c|}{ Cèdre } & \multicolumn{2}{|c|}{ Pin laricio } & \multicolumn{2}{|c|}{ Pin noir } \\
\hline & I & 11 & I & II & I & II \\
\hline $17-08-1983$ & 100 & 82 & & & & \\
\hline $01-09-1983$ & 93 & 36 & 一 & 一 & & \\
\hline $06-09-1983$ & & & & & 96 & 65 \\
\hline $1.5-09-1983$ & 73 & 18 & 63 & 0 & 92 & 60 \\
\hline $29-09-1983$ & 96 & 21 & & & 100 & 71 \\
\hline $12-10-1983$ & 95 & 16 & 70 & 0 & & \\
\hline $13-10-1983$ & & & & & 100 & 15 \\
\hline $28-10-1983$ & & & 33 & 0 & 97 & 31 \\
\hline \multicolumn{7}{|c|}{$\begin{array}{l}\text { I : Plants avec racines blanches à la plantation. } \\
\text { Seedlings planted with white root tips. }\end{array}$} \\
\hline \multicolumn{7}{|c|}{$\begin{array}{l}\text { II : Plants sans racines blanches à la plantation. } \\
\text { Seedlings planted without white root tips. }\end{array}$} \\
\hline
\end{tabular}

Le taux de survie varie de 93 p. 100 à 100 p. 100 pour les plants du traitement I et de 85 p. 100 à 95 p. 100 pour les plants du traitement II. On constate aussi que des plants peuvent survivre sans débourrer.

\subsection{Cas du pin laricio de Corse (fig. 2)}

Après les plantations du $1^{\text {er }}$ septembre et du 15 septembre 1983, les plants du traitement $I(\bullet)$ ont d'une façon générale des potentiels hydriques supérieurs à ceux du traitement II $(\odot)$ qui se caractérisent aussi par une absence de régénération de racines blanches. Pour les deux autres plantations (29 septembre et 12 octobre 1983), les différences de potentiels entre traitements sont moins évidentes. 

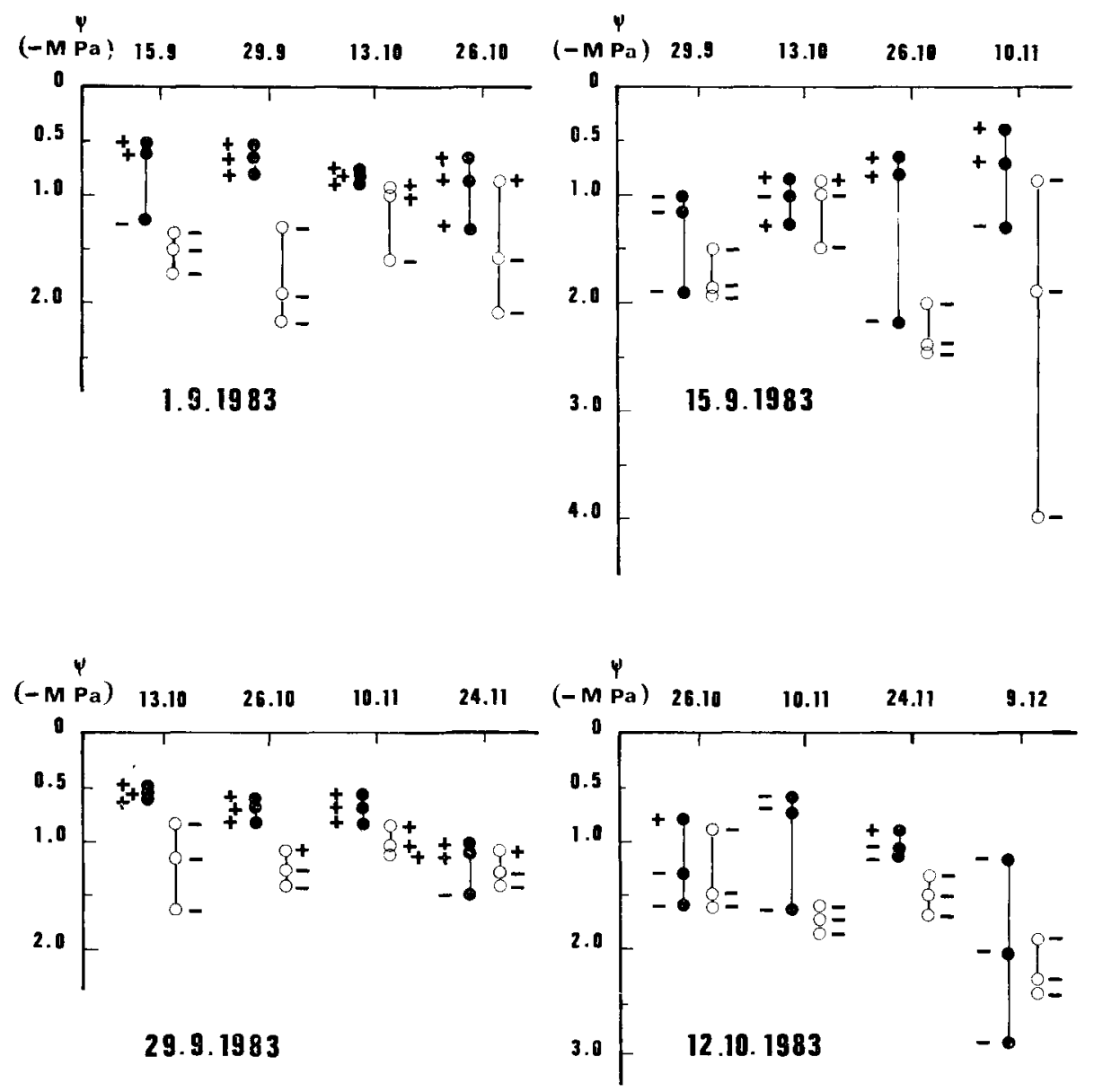

FIG. 2

Evolution, après plantations en automne, du potentiel hydrique de base (-MPa), présence $(+)$ ou absence $(-)$ de racines blanches pour des plants de pin laricio de Corse plantés avec racines blanches $(\bullet)$ et sans racines blanches (O).

Predawn potential after plantation in autumn, presence $(+)$ or absence $(-)$ of white root tips for Corsican pine planted with white root tips $(\bullet)$ and without white root tips $(0)$.

L'effet des gelées du 24 novembre et du 9 décembre 1983 est moins net que chez le cèdre. A cette dernière date, aucun des plants extraits pour mesure ne présentait de régénération de racines blanches.

En juin 1984, le taux de survie variait de 94 p. 100 à 100 p. 100 pour les plants du traitement I et de 84 p. 100 à 100 p. 100 pour les plants du traitement II. La différence essentielle réside dans le taux de débourrement (tabl. 2) qui varie de 33 p. 100 à 70 p. 100 pour les plants du traitement I, alors qu'aucun plant du traitement II issu des différentes plantations n’avait débourré. 


\subsection{Cas du pin noir d'Autriche (fig. 3)}

Après les plantations du 6 septembre, 15 septembre et 29 septembre 1983, les mesures périodiques montrent que les plants du traitement I ont, d'une façon générale, des potentiels hydriques plus élevés que les plants du traitement II qui se caractérisent par une absence fréquente de racines blanches.

Pour les plantations du 13 octobre et du 28 octobre 1983, les potentiels hydriques sont plus proches dans les deux traitements, bien que les plants du traitement II présentent moins souvent des racines blanches.

Au 9 décembre 1983, les racines blanches sont encore présentes (et même plus tard le 26 décembre), alors que cela n'est plus le cas chez le pin laricio et le cèdre.

Les mesures effectuées en juin 1984 ont permis de constater que le taux de survie a été de 100 p. 100 pour les deux traitements. Par contre, des différences notables existent dans les taux de débourrement (tabl. 2).

\section{Tableau 3}

Répartition (p. 100) des plants des différents traitements et especces par classes de potentiel hydrique après les plantations d'automme.

Distribution (p. 100) of seedlings in classes of water potential (predawn potential) after autumn plantations.

\begin{tabular}{|c|c|c|c|c|c|c|c|}
\hline \multirow{3}{*}{ Classes de po } & \multirow{3}{*}{$\begin{array}{l}\text { potentiel hydrique } \\
\text { (MPa) }\end{array}$} & \multicolumn{6}{|c|}{ Espèces et traitements } \\
\hline & & \multicolumn{2}{|c|}{ Cèdre } & \multicolumn{2}{|c|}{$\begin{array}{l}\text { Pin laricio } \\
\text { de Corse }\end{array}$} & \multicolumn{2}{|c|}{ Pin noir } \\
\hline & & I & II & I & II & I & II \\
\hline 0 & $-\quad-0,5$ & 45,1 & 43,1 & 5,1 & & 50,0 & 11,9 \\
\hline$-0,51$ & $--1,0$ & 49,0 & 19,6 & 66,7 & 25,6 & 42,8 & 52,4 \\
\hline$-1,1$ & $--1,5$ & & 19,6 & 15,4 & 30,8 & 7,2 & 21,4 \\
\hline$-1,51$ & $-\quad-2,0$ & 3,9 & 2,0 & 10.2 & 30,8 & & 7,1 \\
\hline$-2,1$ & $-\quad-2,5$ & & 2,0 & 2.6 & 10,2 & & 4,8 \\
\hline$-2,51$ & $-\quad-3,0$ & 2,0 & 3.9 & & & & 2,4 \\
\hline$-3,1$ & $-\quad-3,5$ & & 7,8 & & & & \\
\hline$-3,51$ & - & & 2,0 & & 2,6 & & \\
\hline \multicolumn{8}{|c|}{$\begin{array}{l}\text { I : Plants avec racines blanches à la plantation. } \\
\text { Seedlings planted with white root tips. }\end{array}$} \\
\hline \multicolumn{8}{|c|}{$\begin{array}{l}\text { II : Plants sans racines blanches à la plantation. } \\
\text { Seedlings planted w'thout white root tips. }\end{array}$} \\
\hline
\end{tabular}



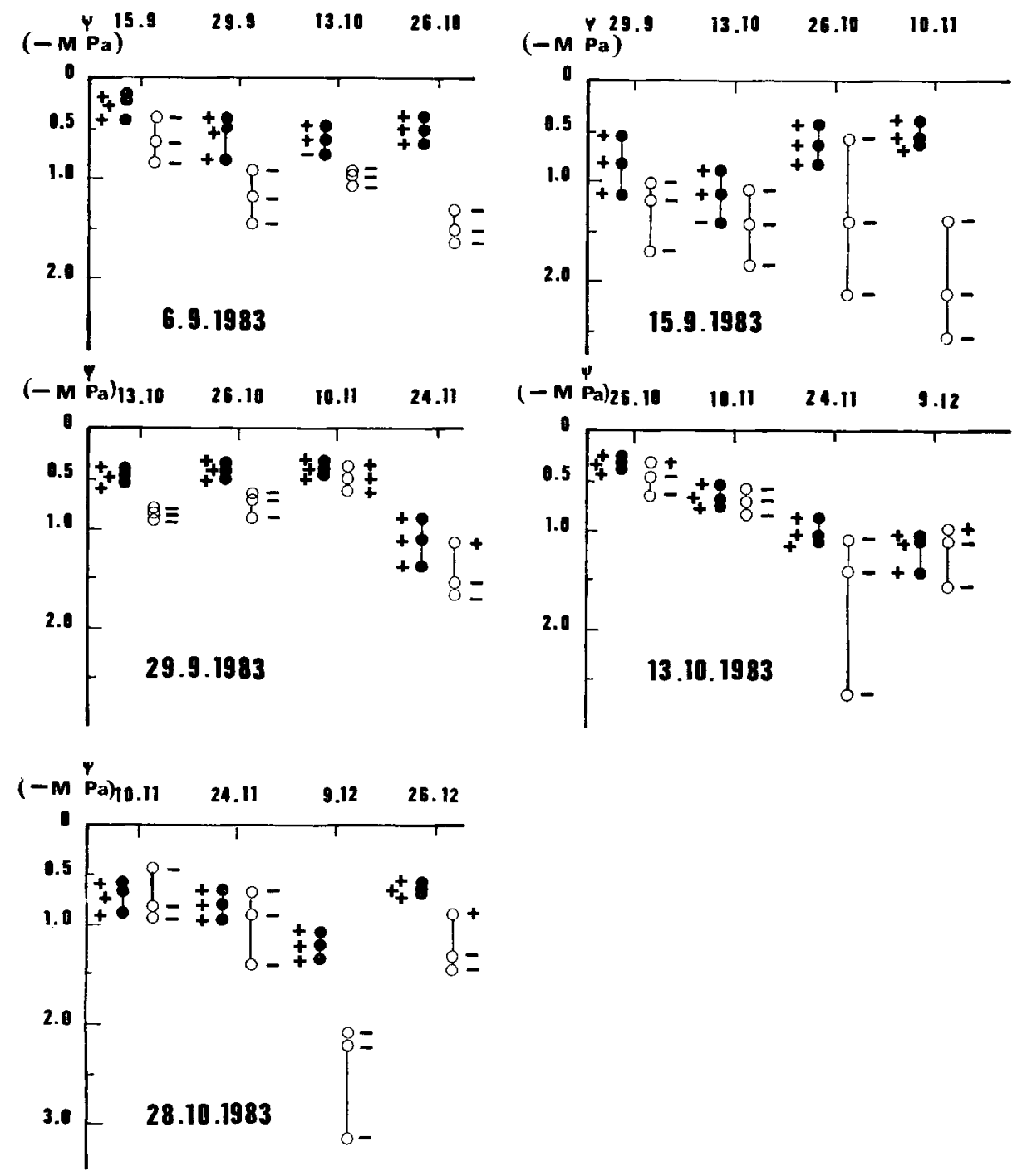

FIG. 3

Evolution, après plantations en automne, du potentiel hydrique de base (-MPa), présence $(+)$ ou absence $(-)$ de racines blanches pour des plants de pin noir d'Autriche plantés avec racines blanches (O) et sans racines blanches (O).

Predawn potential after plantation in autumn, presence (+) or absence (-) of white root tips for Austrian pine planted with white root tips (0) and without white root tips (0). 
D'une façon générale, on constate que les plants avec racines blanches ont un meilleur état hydrique que les autres. Sur le tableau 3, qui représente la répartition par classes de potentiel hydrique des plants étudiés pour l'ensemble des plantations d'automne, on remarque que :

- chez le cèdre, la presque totalité $(94,1$ p. 100) des plants transplantés avec racines blanches n'a pas dépassé un potentiel hydrique inférieur à $-1,0 \mathrm{MPa}$, alors qu'il n'y a seulement que 63 p. 100 des plants du traitement II qui sont dans cette situation ;

- chez le pin laricio de Corse, 71,8 p. 100 des plants du traitement 1 ont eu un potentiel hydrique supérieur à $-1,0 \mathrm{MPa}$, contre $25,6 \mathrm{p}$. 100 pour le traitement II ;

- chez le pin noir d'Autriche, 92,8 p. 100 des plants du traitement I ont un potentiel hydrique supérieur à $-1,0 \mathrm{MPa}$ contre $64,3 \mathrm{p}$. 100 pour le traitement II.

Si on analyse en détail chacun des traitements, on remarque (tabl. 4) que les plants qui, après plantation ont régénéré des racines blanches, ont eu un potentiel hydrique bien meilleur que les autres.

Chez le cèdre et le pin noir d'Autriche, pour les 2 traitements considérés, le potentiel hydrique moyen est voisin de $-0,5 \mathrm{MPa}$. Chez le pin laricio de Corse, il est plus faible $(-0,76 \mathrm{MPa}$ pour le traitement I et $-0,96 \mathrm{MPa}$ pour le traitement II).

\section{TABleau 4}

Moyennes des potentiels hydriques de base (MPa) des plants pourvus ( + ) ou non (-) de racines blanches au monent de la mesure après les plantations d'automne.

Means of water potential (predawn potential) of seedlings with white root tips $(+)$ or without white root tips (-) at the time of measure after autumn plantation.

\begin{tabular}{|c|c|c|c|c|c|c|c|}
\hline \multirow{2}{*}{\multicolumn{2}{|c|}{$\begin{array}{l}\text { Traitements } \\
\text { Présence, absence racines }\end{array}$}} & \multicolumn{6}{|c|}{ Espèce } \\
\hline & & \multicolumn{2}{|c|}{ Cèdre } & \multicolumn{2}{|c|}{$\begin{array}{l}\text { Pin Iaricio } \\
\text { de Corse }\end{array}$} & \multicolumn{2}{|c|}{ Pin noir } \\
\hline \multirow{2}{*}{ I } & + & $-0,475$ & $a^{*}$ & $-0,760$ & $\mathrm{c}$ & $-0,564$ & e \\
\hline & - & $-1,825$ & b & $-1,32$ & d & $-1,05$ & $f$ \\
\hline \multirow{2}{*}{ II } & + & $-0,456$ & $a$ & $-0,955$ & $\mathrm{c}$ & $-0,475$ & $\mathrm{e}$ \\
\hline & - & $-1,817$ & b & $-1,636$ & d & $-1,126$ & f \\
\hline
\end{tabular}

I : Plants avec racines blanches à la plantation. Seedlings planted with white root tips.

II : Plants sans racines blanches à la plantation. Seedlings planted without white root tips.

* Pour chaque espèce, les moyennes sont statistiquement différentes, sauf celles affectées d'une même lettre. 


\subsection{Potentiel hydrique et régénération des racines chez le cèdre et le pin laricio de Corse plantés au printemps}

Immédiatement après la plantation du $1^{\text {'r }}$ avril 1983 , les plants de cèdre du traitement I ont un potentiel hydrique plus élevé que ceux du traitement II. Un mois après, le potentiel hydrique de l'ensemble des plants (tous traitements confondus) atteint des valeurs voisines de - 0,5 MPa. Dès 15 jours après plantation, les plants ont présenté des racines blanches. Pour cette plantation, le taux de survie est de 100 p. 100 pour les 2 traitements.

Pour les pins laricio de Corse, les potentiels restent élevés; voisins de - 0,5 MPa. Les racines blanches sont présentes, quels que soient les traitements considérés. Pour cette espèce, le taux de survie est aussi de 100 p. 100 dans les 2 traitements.

Après la plantation du 17 mai 1983, pour les cèdres, d’une façon générale, les arbres (tabl. 5) du traitement I ont un potentiel hydrique plus élevé que ceux du traitement II.

\section{TABI.EAU 5}

Potentiel hydrique de base $(M P a)$ et présence $(+)$ ou absence $(-)$ de racines blanches chez les plants des traitements $I$ et II après la plantation da 15-5-1983.

Water potential (predawn) (MPa) and presence (+) or absence (-) of white root tips after spring plantation (15-5-1983).

\begin{tabular}{|c|c|c|c|c|c|c|}
\hline \multirow{3}{*}{ Date de mesure } & \multicolumn{5}{|c|}{ Espèce } & \\
\hline & \multicolumn{3}{|c|}{ Pin laricio de Corse } & \multicolumn{3}{|c|}{ Cèdre } \\
\hline & I & II & & I & II & \\
\hline $01-06-1983$ & $\begin{array}{r}-0.66(-) \\
-0.38(+) \\
-0.40(+)\end{array}$ & $\begin{array}{l}-0,7 \\
-0,8 \\
-0,58\end{array}$ & $\begin{array}{l}(+) \\
(+) \\
(+)\end{array}$ & $\begin{array}{l}-0,32(+) \\
-0,38(+ \\
-0,62(+)\end{array}$ & $\begin{array}{l}-0,32 \\
-0,5 \\
-2,9\end{array}$ & $\begin{array}{l}(+) \\
(-) \\
(-)\end{array}$ \\
\hline $15-06-1983$ & $\begin{array}{r}-0,54(+) \\
-0,42(+) \\
-0,38(+)\end{array}$ & $\begin{array}{r}1.4 \\
-1.2 \\
-1.2\end{array}$ & $\begin{array}{l}(-) \\
(+) \\
(+)\end{array}$ & $\begin{array}{r}-0,95(+) \\
-0,38(+) \\
-0,42(+)\end{array}$ & $\begin{array}{l}-3,1 \\
-0,95 \\
-1,15\end{array}$ & $\begin{array}{l}(-) \\
(-) \\
(-)\end{array}$ \\
\hline $29-05-1983$ & $\begin{array}{r}-0.85(+) \\
-0,70(+) \\
-1,30(+)\end{array}$ & $\begin{array}{l}-1.5 \\
-1.2 \\
-1.2\end{array}$ & $\begin{array}{l}(-) \\
(+) \\
(-)\end{array}$ & $\begin{array}{r}-0,34(+) \\
-0,34(+) \\
-0,30(+)\end{array}$ & $\begin{array}{r}-2,60 \\
-0,40 \\
-0,29\end{array}$ & $\begin{array}{l}(-) \\
(+) \\
(-)\end{array}$ \\
\hline $12-07-1933$ & $\begin{array}{r}-0,87(+) \\
-0,80(+) \\
-0,70(+)\end{array}$ & $\begin{array}{l}-0,75 \\
-1,05 \\
-1,15\end{array}$ & $\begin{array}{l}(+) \\
(+) \\
(+)\end{array}$ & $\begin{array}{r}-0,38(+) \\
-0,36(+) \\
-0,31(+)\end{array}$ & $\begin{array}{l}-1,25 \\
-0,45 \\
-0,50\end{array}$ & $\begin{array}{l}(+) \\
(+) \\
(+)\end{array}$ \\
\hline
\end{tabular}

I : Plants avec racines blanches à la plantation. Seedlings planted with white root tips.

Il : Plants sans racines blanches à la plantation. Seedlings planted withont white root tips. 
Le taux de survie atteint 100 p. 100 pour les plants du traitement I contre 80 p. 100 pour le traitement II.

\section{Discussion et conclusion}

Les mesures et les observations effectuées dans ce travail montrent qu'il existe effectivement une relation entre état hydrique des plants après plantation et présence ou absence d'extrémités racinaires blanches.

Les différences entre les potentiels hydriques des plants installés avec leurs extrémités racinaires intactes (traitement $\mathrm{I}$ ) et les potentiels hydriques des plants privés de leurs racines blanches (traitement II) révèlent bien, dès la plantation, le rôle de ces extrémités racinaires en croissance. Ce rôle est encore confirmé par l'amélioration que subit l'état hydrique des plants du traitement II, lorsqu'ils régénèrent de nouvelles racines.

D'une façon générale, il a été constaté que, lorsque le potentiel hydrique de base était inférieur à $-1,5 \mathrm{MPa}$, les plants ne présentaient pas de racines blanches et ceci aussi bien dans le traitement I que dans le traitement II.

Dans quelques cas, il existe des plants qui présentent un potentiel hydrique élevé, malgré l'absence de racines blanches. Ce phénomène ne peut s'expliquer que par une absorption d'eau à partir de racines déjà lignifiées, couplée avec un bon contact dans le sol. Certains plants, malgré une absence totale de régénération racinaire, ont réussi à survivre jusqu'à l'année suivante. Ce rôle des racines lignifiées a été déjà mis en évidence par Kramer \& Bullock (1966) chez Pinus taeda.

L'étude de la répartition des plants par classes de potentiel hydrique (plantations d'automne), fait apparaître des différences nettes entre les trois espèces étudiées :

- pour le traitement I, seulement 5,1 p. 100 des plants de pin laricio de Corse ont eu un potentiel de base compris entre 0 et $-0,5 \mathrm{MPa}$ contre 45,1 p. 100 et 50 p. 100 respectivement chez le cèdre et le pin noir. Ces résultats reflètent les difficultés du pin laricio à maintenir un niveau optimal d'hydratation des tissus, malgré une plantation préservant les extrémités racinaires;

- pour le traitement II, aucun plant de pin laricio de Corse n'a pu conserver un potentiel de base supérieur à $-0,5 \mathrm{MPa}$ contre 43,1 p. 100 de cèdre et 11,9 p. 100 de pin noir. Si l'on prend comme référence - 1,0 MPa, seulement 25,6 p. 100 des plants de pin laricio ont eu un potentiel hydrique supérieur ou égal à ce niveau de sécheresse, contre 62,7 p. 100 pour le cèdre et 64,3 p. 100 pour le pin noir.

Incontestablement, sur ce plan de l'état hydrique des plants après plantation, il y a eu une différence nette entre le pin laricio et les deux autres espèces.

Pour ce qui concerne les taux de survie des plants, il faut constater qu'ils sont importants pour les trois espèces dans les 2 traitements : 100 p. 100 pour le pin noir, ce qui confirme la réputation de très bonne reprise dont bénéficie cette essence, 85 à 100 p. 100 pour le cèdre, 84 à 100 p. 100 pour le pin laricio de Corse. Les pertes observées pour ces 2 dernières espèces confirment leur sensibilité à la plantation à racines nues. Mais, ces taux de survie importants amènent à penser que les fortes 
mortalités notées dans la pratique forestière sont en grande partie la conséquence d'une absence ou d'une insuffisance de précautions après l'extraction des plants de la pépinière. L'arrosage à la plantation constitue aussi très probablement un facteur déterminant.

ANDRÉ (1970) estime que le potentiel de base de - 2,5 MPa constitue, pour l'épicéa et le pin laricio de Corse, un seuil en dessous duquel les plants meurent après plantation. Dans notre travail, les mesures effectuées permettent de penser que ce seuil semble convenir pour le cèdre et le pin noir. Pour le pin laricio, il apparaît que, pour expliquer les mortalités observées, il faut considérer un seuil plus élevé : $-2,0 \mathrm{MPa}$.

Les résultats obtenus dans ce travail confirment ceux de Riedacker \& Arbez (1983) qui avaient trouvé qu'il n'y avait pas de différence dans les capacités de régénération racinaire chez le pin laricio de Corse et le pin noir.

Un autre résultat particulièrement net est l'effet de l'excision des extrémités racinaires blanches sur le débourrement des plants l'année suivante. D’une façon générale (plantations d'automne, pour les 3 espèces étudiées), ce traitement (II) a eu un taux de débourrement inférieur au traitement témoin (I). Dans le cas du pin laricio de Corse, aucun des plants de ce traitement II n'a débourré. Cette espèce présente encore ici une différence importante avec le pin noir et le cèdre. Cette absence de débourrement se traduit par une perte de croissance en hauteur et une baisse de l'assimilation photosynthétique des plants; on sait en effet que la capacité photosynthétique des aiguilles baisse avec l'âge. Ce dernier phénomène peut aussi contribuer à une mortalité l'année suivante.

L'absence ou le faible taux de débourrement est difficile à expliquer. A partir des travaux de différents auteurs (Lavender et al., 1973 ; Arias \& Crabre, 1975 ; in RIEDACKER, 1978), on peut cependant penser que les racines blanches jouent un rôle déterminant pour le débourrement, en fournissant des produits du type gibberelline. Leur absence ou leur nombre réduit aurait pour conséquence de bloquer toute évolution des bourgeons.

Globalement, il apparaît des différences importantes entre les 3 espèces étudićes ici, en défaveur du pin laricio de Corse. Ces différences ne peuvent s'expliquer que par une plus faible régénération racinaire, comme on l'a vu précédemment. Une part importante de l'explication de ces phénomènes pourrait résider dans des dessèchements pendant et après les opérations de plantation en relation avec une régulation moins précoce de la résistance stomatique chez le pin laricio (Aussinac, 1980). Une moindre capacité de prélèvement hydrique par les racines subérisées pourrait aussi intervenir; la résultante des deux phénomènes aboutissant à un plus grand dessèchement des plants. Une moindre tolérance au dessèchement est aussi envisageable si l'on considère - 2,0 $\mathrm{MPa}$ comme seuil fatal pour cette espèce. Pour Pinus radiata, Sands (1984) pense que les conditions de contact entre système racinaire et sol doivent intervenir. Dans le cas des espèces étudiées ici, on ne voit pas a priori dans les conditions de l'expérience comment des différences pourraient exister à ce niveau entre pin noir et pin laricio de Corse.

Reçu le 6 mars 1985. Accepté le 21 aồt 1985. 


\section{Summary \\ Water potential and root regeneration of seedlings of Cedrus atlantica, Corsican pine, Austrian pine after plantation in autumn and spring}

This study deals with the evolution of predawn water potential of cedar (Cedrus atlantica Manctti), Corsican pine (Pinus nigra Arn ssp. laricio poir. var. corsicana) and Austrian black pine (Pinus nigra ssp. nigricans Host) seedlings together with their root development after out-planting with bare-rooted nursery stock in the fall and spring (tabl. 1).

The experiment consisted of two different treatments : intact ;

- treatment I : plantations were realised with seedlings having their white root tips

- treatment II : where all white roots were pruned and seedlings were replanted immediately under field conditions together with the seedlings of treatment $I$.

The results obtained in both treatments showed a direct relationship between seedlings water potential after plantation and the existence of white root extremites. Seedlings presenting white roots at the moment of measurement, had higher (less negativc) water potential than seedlings without white roots (fig. 1, 2, 3; tabl. 4 and 5). In general, it was observed that, in plants of both treatments with water potential inferior to - $1.5 \mathrm{MPa}$ did not present white roots. The assessment of the seedlings water potential (tabl. 3) showed clear differences between the three species. In plants subjected to treatment I, 5.1 p. 100 of Corsican pine seedlings had a water potential between zero and $-0.5 \mathrm{MPa}$ against 45.1 p. 100 and 50 p. 100 respectively for cedar and Austrian black pine seedling. For seedlings subjected to treatment II, all Corsican pine seedlings showed a water potential inferior to $-0.5 \mathrm{MPa}$ whereas $43.1 \mathrm{p} .100$ of cedar and 11.9 p. 100 of Austrian black pine were found with a water potential superior to $-0.5 \mathrm{MPa}$. However, seedling survival was excellent for the different species. On the otherhand, the removal of white roots (treatment II) from seedlings of cedar and Austrian black pine at plantation in autumn, lowered their budbreak rate in the following spring compared with seedlings of treatment $\mathrm{I}$. Budburst did not occur in Corsican pine seedlings subjected to treatment II in autumn (tabl. 2).

The apparent differences between the tree species with Corsican pine seedlings being most deleteriously, affected by pruning of white roots at plantation, reveal that, seedlings of this specie are less efficient in maintaining high levels of hydration as a consequence to their failure in regulating their water loss, in low ability of their subrized roots to absorb water and/or to their less tolerance to dryness.

Key words : Predawn potential, root regeneration, plantation.

\section{Références bibliographiques}

ANDRÉ P., 1970. Détermination de l'état physiologique des plants forestiers. R.F.F., XXXI, $6,465-472$.

Arias A., Crabbe J., 1975. Les gradients morphogénétiques du ramcau d'un an des végétaux ligneux en repos apparent. Données complémentaires fournies par l'ćtude de Prunus avium L. Physiol. Vég., 13 (1), 69-81.

Arbez M., 1971. Croissance des racines du pin laricio de Corse ( $P$. nigra Arn. ssp. Laricio) au stade juvénile. Ann. Sci. For., 28 (3), 259-288.

Aussenac G., 1980. Comportement hydrique de rameaux excisés de quelques espèces de sapins et de pins noirs en phase de dessication. Ann. Sci. For., 37 (3), 201-215.

Aussenac G., Granier A., Ibrahim M., 1984. Influence du dessèchement du sol sur le fonctionnement hydrique et la croissance du douglas [Pseudotsuga menziesii (Mirb.) Franco]. Ecol. Plant., 5 (19), 3, 241-253. 
EL Nour M., 1984. Etude de la croissance et de la régénération des racines du chêne pédonculé, du cèdre, du pin laricio de Corse et du pin noir. Essais d'amélioration de la reprise après plantation à partir de modification du fonctionnement racinaire. Thèse Nancy I et I.N.R.A., 118 p.

Kramer P., Bullock H., 1967. Scasonal variation in the proportion of suberized and unsuberized roots of trees in relation to the absorption of water. Am. J. Bot., 53. 200-204.

Lavender D.P., Swiet G.B., Zaerr J.B., Hermann R.K., 1973. Spring shoot growth in Douglas fir may be initiated by Gibberelins Exported from the roots. Sciences, 181, 838-839.

Riedacker A., 1978. Régénération et croissance de la partie souterraine ct aérienne de cèdres placés sous climat constant. Ann. Sci. For., 35 (2), 117-138.

Riedacker A., Arbliz M., 1983. Croissance et régénération des racines des semis de pin laricio de Corse et de pin noir en chambre climatisée et in situ. Ann. Sci. For., 40 (1), $79-110$.

Sands R., 1984. Transplanting stress in Radiata Pine. Austr. For. Res., 14, 67-72. 